\title{
Complete Trisomy 18 Syndrome
}

National Cancer Institute

\section{Source}

National Cancer Institute. Complete Trisomy 18 Syndrome. NCI Thesaurus. Code C101362.

A constellation of symptoms that occur as a result of the presence of a complete third copy of the 18th chromosome. Characteristics include profound mental retardation and severe malformations. Individuals with this syndrome rarely live past one year. 